\title{
Trajectories of Informal Care Intensity among the Oldest-Old Chinese
}

Dr. Bo Hu

8.01 Pankhurst House, Clement's Inn

Care Policy and Evaluation Centre (CPEC), Department of Health Policy London School of Economics and Political Science

London WC2A 2AZ, UK

b.hu@1se.ac.uk 


\begin{abstract}
Countries around the world face increasing demand for long-term care in the older population. Yet, the longitudinal patterns of long-term care use and the underlying predictors have not been well understood, which impedes efficient care planning and timely service delivery. This study investigates the trajectories of informal care intensity in the oldest-old Chinese population and identifies the most important predictors of care trajectories. The data come from four waves of the Chinese Longitudinal Health Longevity Survey (CLHLS 2005-2014, N=10,292). We conducted the latent trajectory analysis (LTA) to cluster people's diverse trajectories into a finite number of groups. We built machine learning (ML) models to predict people's care trajectories and ranked the relative importance of the predictors.
\end{abstract}

The LTA identified three distinct trajectories of informal care intensity: the low, increased and high intensity trajectories. Care intensity increases in all three trajectories. Older people with more severe limitations, females, urban residents, people with a higher income, and people with more daughters in the first wave are more likely to follow the increased or high intensity trajectory rather than the low intensity trajectory in the following decade. The random forest classifier has the best overall prediction performance among the four machine learning models. Its prediction accuracy can be further improved via model optimisation. Oldest-old people in China follow divergent trajectories of care utilisation, and inequality of informal care intensity is discernible across time, demonstrating the need for timely and targeted delivery of government support to those who need it most. Accurate prediction of care trajectories will be of great value to policy makers and practitioners in relation to the planning of personalised care and the equitable allocation of care resources.

Keywords: Informal care intensity, care inequality, oldest-old Chinese, latent trajectory analysis, machine learning 


\section{Introduction}

Long-term care is crucial to the wellbeing of older people who are experiencing, or at risk of a significant loss of intrinsic capacity. It helps older people maintain a level of functional ability consistent with their basic rights, fundamental freedoms, and human dignity (World Health Organisation, 2015). Population ageing is a global issue. As the number of older people continues to increase in the coming decades, the demand for long-term care is set to rise substantially.

China is experiencing unprecedented population ageing. At present, there are 250 million older people aged over 60 . It is projected that, by 2040 , the number of older people will increase by $73 \%$, whereas the number of younger adults aged 20-59 will decrease by $14 \%$ (United Nations, 2019). Unlike many high-income countries, China still relies heavily on informal care to meet older people's care needs (Yang et al., 2016). While the Chinese government has started investing in community-based formal care and policies relating to care provision have been put in place, utilisation of these services is still the exception rather than the norm in many parts of the country. It takes time to train the workforce and improve the overall quality of formal care, and preferences for informal care are unlikely to change drastically in the short-run (Shi \& Hu, 2020; Wang et al., 2020).

Oldest-old people, namely those aged over 80 or 85 , are the fastest-growing group in the older population. Their support networks reduce with age, yet they are the most intensive users of long-term care (Feng et al., 2012). In the case of China, an overwhelming majority of them are widowed, so children or grandchildren become their main or sole caregivers, which creates further uncertainty regarding the adequacy of existing resources to meet people's care needs (Zhu, 2015). It has been estimated that around $50 \%$ of Chinese people in this age group have unmet or undermet care needs (Peng et al., 2015). In this context, the sustainability of the long-term care system and the minimisation of the risk of unmet needs are the two paramount objectives of the Chinese government. The fulfilment of these objectives, however, entails careful planning guided by a thorough understanding of the existing patterns and predictors of care utilisation. 
This study examines the hours of informal long-term care (i.e. informal care intensity) that oldest-old people in China receive from their children or grandchildren. Drawing on longitudinal data spanning a decade, it aims to map out the trajectories of care intensity and identify the most important predictors associated with the trajectory memberships.

\section{Predictors of informal care utilisation}

A well-established body of literature has examined the predictors of informal care use or intensity. The behavioural model of care utilisation has been widely used to guide the analyses (Anderson \& Newman, 2005). According to this framework, the use of informal care is driven by need factors, predisposing factors, and enabling factors. Need is the most immediate reason for using long-term care. Older people with more severe functional limitations or lower physical capacity are more likely to receive informal care (Kemper, 1992; Pickard et al., 2000; Suanet et al., 2012; Vlachantoni et al., 2015) or receive more hours of informal care each month (Hu \& Ma, 2018; Wolf, 2014).

The predisposing factors are the personal characteristics that affect the propensity of care use prior to the onset of care needs. The important predisposing factors vary from one country to another. SoleAuro and Crimmins (2014) found that gender is an important predictor of informal care use in England and the US, but not in Spain. Both the likelihood of using informal care and the intensity of informal care differ markedly according to ethnicity in the US (Weiss et al., 2005). Wang et al. (2020) reported that age is positively associated with the probability of receiving informal care from children.

Enabling factors are the resources, means, and instruments that facilitate care use. Availability and proximity of support networks enable access to informal care. Older people who are married or have more children are more likely to be users of informal care (Grundy \& Read, 2012; Kemper, 1992). Wolf (2014) reported that older people receive more hours of informal care if they have more unmarried children. Furthermore, working-age adults with a higher paid job or higher education have a lower propensity to give up employment and provide care, which results in a reduced support network for older people with care needs (Carmichael \& Charles, 2003; Hanaoka \& Norton, 2008). 
Most of the existing studies on informal care intensity are based on analyses of cross-sectional data, which provide a snapshot of the patterns of care use. Only a few studies have investigated the longitudinal patterns so far. Miller and McFall (1991) found that the intensity of informal care for older people was stable over a two-year period and that changes in intensity occurred in response to changes in people's functional status. Li (2005) reported that the number of weekly hours of informal care received by older people in the US decreases markedly in the two years after the provision of public-funded home care, which indicates a substitutive relationship between formal and informal care.

Two features can be noted in the existing research on informal care utilisation. First, most studies assume that the hours of informal care received by older people have a homogenous distribution, but rarely do they consider the possibility that they may come from qualitatively distinct distributions. The latter point means that recipients of informal care may belong to different latent groups and their care intensity may follow divergent trends.

Second, many of the existing studies have focused on the explanatory or associative relationship between a particular factor and care utilisation, but less effort has been devoted to the prediction performance of these predictors as a whole. Yet, predictive analytics is vitally important from the perspective of care planning and resource allocation. First, it provides valuable information about the predicted level of care consumption given a person's characteristics. Aggregating the prediction of each individual leads to predicted care utilisation at the population level (Wittenberg et al., 2020). Second, if structural changes are expected in the population (e.g. a demographic shift or a rise in the socioeconomic profiles), predictions can be made about the likely changes in the care resources being required, which provides guidance for the government to plan ahead and make preparations.

In this study, we use the machine learning (ML) approach to investigate the predictors of informal care intensity. Many widely used machine learning models are non-linear non-parametric by construction and can automatically capture the complex relationships between variables, which provides a time-efficient way to improve prediction accuracy and inform decision making on resource allocation (Casanova et al., 2018). 


\section{Research methods}

Data

The data come from the Chinese Longitudinal Health Longevity Survey (CLHLS). The survey collected ageing and care-related information from a random sample of the oldest-old people in 23 provinces across China. The sampling areas covered $85 \%$ of the Chinese population (Zeng et al., 2002). The baseline survey took place in 1998 , with follow-up surveys conducted in 2000,2002 , 2005, 2008, 2011 and 2014. The CLHLS study was approved by the Research Ethics Committee of Peking University (IRB00001052-13074), and all participants provided written informed consent.

Our study draws on the four most recent waves of the CLHLS data. It follows a cohort of 10,292 older people who were aged 80 and over and living in private households from 2005 to 2014 . Such a study design makes sure that there is a sufficiently large sample for analysis throughout the study period. As shown in Figure 1, the attrition was substantial in the follow-up surveys. By 2014, only 674 people were still in the survey. The other people had either moved to a care home or died, or were lost to follow up.

\section{Measurements}

Our key outcome variable of interest is informal care intensity. In each of the four waves, the survey asked each respondent how many hours their children and grandchildren had helped them with activities of daily living (ADLs) in the previous week. We created a care intensity variable for each wave, with more hours of care indicating a higher level of care intensity. These four continuous variables, namely care intensity in waves 1 to 4 , were prepared for later analyses. The survey did not ask respondents whether they had received help with instrumental activities of daily living (IADLs) or for how many hours children and grandchildren had helped them with IADL tasks.

Guided by the theoretical framework discussed in the previous section, we examined the need, demographic, and socioeconomic factors which might be associated with care utilisation. For the need factors, we examined people's functional capability and morbidity. The survey asked respondents about their ability to perform six ADL tasks: bathing, dressing, toileting, indoor mobility, continence, 
and eating. The ability to perform each task was measured on a three-point scale: $0=$ no assistance, $1=$ requiring some assistance, and $2=$ requiring complete assistance. By adding up the scores and dividing the total score by 6 , we created a measure of severity of ADL disability that ranges from 0 (no ADL disability) to 2 (severe ADL disability).

The survey included five IADL tasks: visiting a neighbour, shopping, cooking, washing clothes and taking public transport. The ability to perform each task was measured on a three-point scale: $0=$ no help, $1=\mathrm{I}$ need some help, and 2=I cannot do it. By adding up the scores and dividing the total score by 5 , we created a measure of severity of IADL disability, which ranges from 0 (no ADL disability) to 2 (severe ADL disability). There are alternative approaches to measuring functional capability such as calculating the latent scores of ADL or IADL disability on the basis of an item response model or a latent factor model. The analyses conducted by Spector and Fleishman (1998) demonstrated that the 'raw count' scores of ADLs and IADLs are good approximations of latent scores derived from those models.

The survey asked respondents whether they had any of the following 15 chronic diseases: hypertension, diabetes, heart disease, stroke, bronchitis or asthma, tuberculosis, cataract, glaucoma, cancer, prostate tuber, gastric ulcer, Parkinson's disease, bedsore, arthritis, and dementia. Similar to the ADL and IADL limitations, we created a continuous variable by counting the number of chronic diseases reported by each respondent.

For the demographic factors, we examined age, gender, and rural-urban residence. The age variable has three categories: $80-89,90-99$, and more than 100 years old. The residence variable was dichotomised. Older people living in urban China were coded as 1, and those in rural China were coded as 0 . For socioeconomic status, we examined people's self-report economic status, annual household income and years of schooling. The survey asked respondents to rate their economic status compared to others living in the same area on a five-point scale: very rich, rich, average, poor, and very poor. Based on this question, we created a variable with three categories $(0=$ poor, $1=$ average, and $2=$ rich). Annual household income is a continuous variable. We logarithmically transformed the income variable so that the variable was approximately normally distributed. Based on people's years 
of schooling, we created a dichotomised variable: $0=$ no formal education and $1=$ receipt of formal education. For the enabling factors, we examined three predictors: living arrangements, the number of living sons, and the number of living daughters. The living arrangements variable has three categories: living with their children or grandchildren, living with their spouse only, and living alone or with other family members. The variable related to living sons (daughters) also has three categories: no son (daughter), 1-3 sons (daughters), and more than 4 sons (daughters).

We analysed the primary caregivers of older people, given the substitutional relationships between different caregivers (Hanaoka \& Norton, 2008). The survey asked respondents to report their primary caregivers in regard to their ADL limitations. We created a variable with three categories: no ADL limitations or not receiving care, having their (grand)children or spouse as their primary caregivers, and having other relatives or paid helpers as their primary caregivers.

\section{Statistical analysis}

The statistical analysis consisted of two steps. In the first step, we conducted the latent trajectories analysis to cluster the trajectories of informal care intensity into groups. The latent trajectory model assumes that the population distribution of the trajectories is a finite mixture of $\mathrm{J}$ distributions, which can be written as:

$$
P\left(\boldsymbol{C}_{\boldsymbol{i}} \mid t\right)=\sum_{j=1}^{J}\left[\pi_{j} \times P\left(\boldsymbol{C}_{\boldsymbol{i}} \mid t, j\right)\right]
$$

Where $\boldsymbol{C}_{\boldsymbol{i}}$ denotes the longitudinal sequence of care intensity for individual i, $t$ denotes the time variable ( $t=1-4), \pi_{j}$ denotes the probability of belonging to a trajectory $\mathrm{j}$, and $P\left(\boldsymbol{C}_{\boldsymbol{i}} \mid t, j\right)$ denotes the probability of $\boldsymbol{C}_{\boldsymbol{i}}$ given trajectory j. There is substantial attrition in the follow-up surveys of the CLHLS (figure 1). We suspect that people in different trajectories of informal care intensity may have different probabilities of dropping out. To account for the non-random attrition, we followed the specification by Haviland et al. (2011) and Zimmer et al. (2012) and included a dropout parameter which affects the conditional probability of $\boldsymbol{C}_{\boldsymbol{i}}$ :

$$
P\left(\boldsymbol{C}_{\boldsymbol{i}} \mid t, j ; \theta_{\tau}^{j}\right)=\left[\prod_{t=1}^{\tau_{i}-1} p\left(c_{i t} \mid w_{i t}=0, t, j\right)\left(1-\theta_{t}^{j}\right)\right] \theta_{\tau_{i}}^{j}
$$


Where $w_{i t}=1$ if individual i dropped out by $\mathrm{t}$ and $w_{i t}=0$ otherwise. $\tau_{i}$ denotes the wave when individual i dropped out $(\tau=2,3$ or 4$) . \theta_{\tau_{i}}^{j}$ denotes the probability of individual i dropping out in wave $\tau_{i}$ given trajectory $\mathrm{j}$. We estimated $\theta_{\tau_{i}}^{j}$ using a logistic regression where the predictors of dropout were informal care intensity in the previous wave and personal characteristics (including age, ADL score, and IADL score) in 2005. $c_{i t}$ denotes the weekly hours of informal care received in wave $t$ for individual $\mathrm{i}$. We assume that $c_{i t}$ has a censored normal distribution and is linked to a polynomial function of the time variable of the following form:

$$
c_{i t}^{*}=\beta_{0}^{j}+\beta_{1}^{j} \times \lambda_{t}+\beta_{2}^{j} \times \lambda_{t}^{2}+\varepsilon_{i t}
$$

Where $c_{i t}=c_{i t}^{*}$ if $0 \leq c_{i t}^{*} \leq 168, c_{i t}=0$ if $c_{i t}^{*}<0$, and $c_{i t}=168$ if $c_{i t}^{*}>168$. Some older people may not receive any informal care, while others may receive around-the-clock care that amounts to 168 hours per week at the maximum. $\beta_{0}^{j}, \beta_{2}^{j}$, and $\beta_{2}^{j}$ are the coefficients that determine the shape of the polynomial and $\varepsilon_{i t}$ is the error term.

Specifying different values for the grouping parameter $\mathrm{J}$ results in competing models. We chose the model with the largest BIC score. For the selected model, we calculated the average posterior probability (APP) and the odds of correct classification (OCC) for each trajectory to evaluate the model's adequacy. According to Nagin (2005), APP should be at least 0.7 and OCC should be at least 5 for all groups. We created a categorical variable that grouped older people according to their membership of the different trajectories. This was used as the outcome variable in the second step of the analysis. Stata v.15 was used to conduct the analysis (syntax: traj)

In the second step, we used the machine learning (ML) approach to investigate whether the baseline predictors could accurately predict people's trajectories of informal care intensity. We converted the multi-categorical variable of care trajectories into a series of binary outcome variables and conducted pair-wise classifications. The execution of the ML approach involves splitting the dataset into a training set and a validation set (Hastie et al., 2009). A prediction model is trained on the training set, and its prediction accuracy is tested on the validation set. Such a procedure, known as crossvalidation, is used to detect over-fitting of the prediction model (Facal et al., 2019). In the base case 
analysis, we randomly selected $70 \%$ of the sample as the training set and treated the rest $(30 \%)$ of the sample as the validation set. A severe imbalance of observations in the outcome variable (see below) leads to ML models with almost perfect specificity (true negative rate) and poor sensitivity (true positive rate). Such a model is of limited use for care planning and resource allocation because it predicts poorly the more intensive trajectories of informal care. We used the down-sampling approach to remedy this issue (Kuhn \& Johnson, 2013).

We trained four different classification models (i.e. classifiers) on the training set: the binary logistic regression model (LR), random forests (RF), support vector machines (SVM) and artificial neural networks (ANN), and compared their prediction performance on the validation set. We focused on three indicators of prediction performance: sensitivity, specificity, and accuracy (weighted average of sensitivity and specificity). We used the default values of the tuning parameters (i.e. hyperparameters) in the base case analysis. For the RF classifier, we further calculated the relative importance of the predictors according to their contribution to prediction accuracy. Their relative importance was normalised on a scale from 0 (least important) to 100 (most important).

We conducted further analyses to improve the prediction performance of the machine learning models. First, we recalculated the prediction accuracy based on the 10-fold cross-validation (Facal et al., 2019) and the bootstrap (Casanova et al., 2018), respectively. Second, we used the grid search technique to look for hyperparameters that optimised the prediction models. Apart from the income variable, which has $10 \%$ missing values, all of the other variables have less than $1 \%$ missing values. The analyses were conducted on completed cases. $R$ version 3.6.1 was used to conduct the analysis ( $R$ package: caret).

\section{Results}

In $2005,3,094$ out of 10,292 people received informal care from (grand)children in relation to their ADL limitations. On average, they received 44 hours of care per week. The distribution of care intensity is multimodal, which confirmed our rationale for conducting latent trajectory analysis (see Figure A1 in appendix). We specified four competing latent trajectory models (Figure 1). The BIC 
scores indicate that the three-group model has the best fit. In this model, the intensity of informal care followed three distinct trajectories: low intensity, increased intensity, and high intensity.

Among the 10,292 people in $2005,85.8 \%(n=8,834)$ followed the low intensity trajectory, $10.0 \%$ $(\mathrm{n}=1,027)$ followed the increased intensity trajectory, and $4.2 \%(\mathrm{n}=431)$ followed the high intensity trajectory (Table 1). People in the low intensity trajectory on average received 6.8 hours of informal care in $2005,1.5$ hours in 2008, 5.2 hours in 2011, and 8.4 hours in 2014. For people in the increased intensity trajectory, the average number of hours of informal care received rose from 29.1 hours in 2005 to 107.5 hours in 2014 . People in the high intensity trajectory on average received 121.4 hours of informal care in 2005, which rose to 165.3 hours in 2008 and 168 hours in 2011 . The diagnostic statistics indicate that the three-group model is adequate in terms of identifying and clustering latent groups (APP $>0.7$ and $\mathrm{OCC}>5$ ).

The attrition analysis in the latent trajectory model shows that the probability of attrition is positively associated with care intensity in the previous wave, age, ADL limitations, and IADL limitations in the first wave (Table 1). People in the three trajectories had markedly different probabilities of attrition. None of the care recipients in the high intensity trajectory was still participating in the survey in wave 4. The attrition rates between waves 1 and 4 were $83.0 \%$ in the increased intensity trajectory and $17.0 \%$ in the low intensity trajectory, respectively. These results highlight the importance of accounting for non-random attrition in the latent trajectory model to avoid the incorrect clustering of individuals.

Table 2 shows the sample characteristics in 2005 and the univariate association between each predictor and the care trajectory variable. Most of the predictors under investigation are significantly associated with the memberships of care trajectories. People in the high intensity trajectory on average had an ADL score of 0.89, while the average scores of people in the low and increased intensity trajectories were 0.20 and 0.41 , respectively. People in the high intensity trajectory had a significantly higher level of care needs in 2005. In regard to enabling factors, living arrangements are significantly associated with the trajectories of care intensity. Among people in the high intensity trajectory, $91 \%$ were living with their (grand)children in 2005 , in contrast to $72 \%$ among people in the low intensity 
trajectory. People in the increased or high intensity trajectories had more daughters than those in the low intensity trajectory, but the number of sons did not differ significantly. In relation to demographic factors, people in the increased or high intensity trajectories were more likely to be females, belong to a higher age group or live in urban China. In terms of socioeconomic profiles, people in the increased or high intensity trajectories had a higher income or had received a better education than people in the low intensity trajectory. For people in the high intensity trajectory, $78 \%$ of them had (grand)children and $3 \%$ had a spouse as their primary caregiver in 2005 , whereas the proportions were $23 \%$ and $2 \%$, respectively, for people in the low intensity trajectory.

For the classification between the increased and low intensity trajectories, the random forest classifier has the best overall performance, with the prediction accuracy reaching 0.64 (Table 2). The neural network classifier reports a much higher level of sensitivity (0.72), but its specificity is poor (0.54). For the classification between the high and low intensity trajectories, all of the models report a prediction accuracy of 0.79 . The sensitivity reported by the support vector machine classifier is the highest (0.84) among the four classifiers, but its specificity is also the lowest $(0.73)$. For the classification between the high and increased intensity trajectories, the RF and SVM classifiers report the highest accuracy (0.69). The RF classifier reports a higher level of sensitivity (0.71) than the SVM classifier, but the specificity is lower (0.66).

The prediction accuracy of the RF classifier can be further improved by using more robust sampling methods and fine-tuning the hyperparameters. If we combine the 10 -fold cross-validation with the optimised hyperparameters, the prediction accuracy reaches $0.65,0.81$, and 071 , respectively, in the three pair-wise classifications (Table 3). This represents a $2 \%$ improvement in accuracy compared to the RF classifier and a 3\% improvement compared to the LR classifier in the base case analysis.

ADL and IADL limitations in 2005 are consistently the most important predictors across all trajectories (Table 4). All of the classification models rank age and rural-urban residence as moderately important predictors. Having other people as their primary caregivers, having more sons, a high level of education, and poor self-reported economic status are consistently the least important predictors across all trajectories. The relative importance of other predictors differs according to the 
trajectories under investigation. Having (grand)children/spouse as their caregivers, more chronic diseases, and gender are the third to fifth most important predictors in the classification between the increased and low intensity trajectories. For the classification between the high and low intensity trajectories, living arrangements and income follow functional limitations as the next most important predictors. Income is the second most important predictor in the classification between high and increased intensity.

\section{Discussion}

This study used longitudinal data from a national survey to map the trajectories of informal care received by Chinese older people aged over 80 . The analysis focused on care provided by children and grandchildren because most Chinese people in this age group are widowed and do not have access to spouse care. In the CLHLS dataset, a tiny proportion of people (1.9\%) reported that their spouse was their primary caregiver.

We found considerable heterogeneity in people's care journeys in later life. The LTA clustered the diverse care trajectories into three distinct groups, all of which saw an increase in the number of hours of informal care received over a decade. The model estimated that the proportions of people following the low, increased and high intensity trajectories were $86 \%, 10 \%$ and $4 \%$, respectively. The number of older people aged over 80 in China has reached 27 million (United Nations, 2019), which translates into 2.7 million people in the increased intensity trajectory and 1.1 million people in the high intensity trajectory.

Our findings confirm the usefulness of the behavioural model of care utilisation in longitudinal research. Guided by this framework, we identified a comprehensive list of factors in the baseline survey that are significantly associated with trajectories of informal care intensity. Previous studies based on cross-sectional datasets have reported that informal care intensity increases with the level of care needs, which is consistent with our results. Moreover, our analysis extends the existing knowledge by showing that a higher level of care needs is positively associated with the probability of staying in the increased or high intensity trajectories over time. Such a finding has important 
implications for the Chinese long-term care system. Older people's need for care develops with age. The United Nations (2019) has projected that the average life expectancy of the Chinese older population will continue to rise in the following decades, so we expect to see a parallel increase in the overall level of care needs in the older population. This implies that not only will there be more older people needing care, but there will also be a higher proportion of older people needing more intensive care for a sustained period of time.

Unmet needs result from care receipt not keeping up with an increase in care needs ( $\mathrm{Hu} \& \mathrm{Li}, 2020)$. Previous studies have systematically investigated the presence, intensity, and determinants of unmet care needs in the older population (Burchardt et al., 2018; Hu \& Wang, 2019; Vlachantoni, 2019; Zhu, 2015). In comparison, little is known about the duration and trajectories of unmet needs. Some older people may experience substantial unmet needs for several years, while others may experience rising or decreasing unmet needs from one year to another, all of which may have profound consequences for their health and wellbeing. They are not the focus of this study, but the trajectory analysis of care receipt is a crucial step preceding further investigations into those issues.

Our study has found great inequality in the distribution of informal care resources among Chinese older people aged over 80 . Females, urban residents, and people with better education receive more hours of care from their (grand)children. Meanwhile, we found a strong gendered division in caregiving behaviour. Daughters or daughters-in-law in Chinese society are expected to be the main care providers within a family (Zhan \& Montgomery, 2003). Our analysis has shown that having more daughters is positively associated with more hours of informal care. However, having more sons makes little difference. This finding is consistent with the results reported by Grundy and Read (2012) and Robards et al. (2015), although those studies focus on older people in England.

Studies in European countries have reported that informal care utilisation is pro-poor, which means that informal care is more concentrated among older people with a lower income (García-Gómez et al., 2015; Rodrigues et al., 2018). In our study, income is also an important predictor, but the care trajectories are pro-rich: people in the increased or high intensity trajectories have a higher household income. The positive relationships between care intensity and income remain significant even after we 
control for care needs (full results available upon request). We think that the reasons for this are threefold. First, people's preferred sources of care are insensitive to their financial status. The overwhelming majority of older people prefer informal to formal care regardless of their income (Shi $\& \mathrm{Hu}, 2020)$. Second, accessibility to formal care is limited and unbalanced in China. While richer families in large Chinese cities may hire paid caregivers to meet their care needs, most people in less developed cities or in rural areas have no access to caregivers from outside the family even when they can afford those services. This is different from the case of developed countries, where richer families have the option of substituting informal care with formal care (Li, 2005; Van Houtven \& Norton, 2004). Finally, the pension age in China is 60 for men and 50-55 for women (Liu \& Sun, 2016). For older people aged 80 and over, most of their children are either retired or close to retirement. If the household income is higher, children can afford to scale down their engagement in economic activities and spend more time providing care for their parents.

The predicted increase in care demand and the inequality of care utilisation in the older population call for continued government interventions. Formal care and support should be delivered to those who need them most, in a fashion that upholds social justice and distributive fairness. One objective of this study is to build prediction models that can be used to accurately predict the trajectory membership of an older person in the future based on his or her observed characteristics at present. Although our study focuses on the predicted trajectories of informal care in the oldest old population in China, the same methodology used here applies to the prediction of the trajectories of formal care for older people in general or those with specific health conditions such as stroke or dementia in other countries.

Well-designed prediction models can play a significant role in the timely delivery of services and the equitable allocation of care resources. In many countries, the provision of government support is based on the assessment of a person's observed characteristics, such as their level of care needs and the availability of family support (Colombo et al., 2011). The Chinese government also plans to develop such an assessment system as the basis for providing community care (Hu et al., 2020). The provision of government support, however, does not take place instantly. In practice, assessing care 
needs, making decisions on the level of support, securing funding, and delivering services all take time. By the time the assessments are finished, and care resources are made available, an individual's care needs may have already changed. Predicted care trajectories provide useful information about the required care resources for individual clients in the future. Guided by such information, policy makers or practitioners can secure the funding or resources and plan the provision of care in advance. This is especially important for those individuals who are predicted to follow the high or increased intensity trajectories because they may need government support more urgently than others. When the care needs of an individual do rise as predicted, the planned or reserved resources can be mobilised, and care can be delivered to the person more swiftly. Timely support is as important as adequate support.

The ML approach proves to be a useful tool to predict care trajectories. For the classification between the low and high intensity trajectories, the predictors lead to correct predictions in more than $80 \%$ of cases. The prediction accuracy is comparatively lower for the classification between the low and increased intensity trajectories $(65 \%)$. One plausible explanation is that people in these two trajectories started with a similarly low level of care intensity in 2005. It was not until later waves that the divergence of trajectories emerged. A future investigation of the factors associated with older people's inter-temporal transitions in terms of functional limitations and support networks may help to further improve the model performance.

Among the four ML models, the RF classifier has the best prediction performance. The prediction accuracy of the optimised RF classifier is 3\% higher than that of the LR classifier. To understand the significance of this result, it is important to keep in mind that there are 27 million older people aged over 80 in China. Every $1 \%$ increase in prediction accuracy can potentially lead to a better targeted allocation or better planning of care resources for 0.3 million people. For a country with a massive population, even incremental improvements in the long-term care system can have a large impact on numerous people's lives.

The limitations of this study should be noted. First, in terms of the enabling factors, we only examined living arrangements and the number of children. Due to data availability, we were not able to look at other characteristics of children or grandchildren, such as employment status, health conditions and 
child-rearing responsibilities, which may also affect people's caregiving capacity and further improve the performance of the prediction models. Second, our analysis focused on the ADL-related care provided to the oldest-old population in China. Despite the generalisability of our methodology, the actual trends of care utilisation and their predictors may be different for other groups of care users, such as those with IADL limitations only or older people with dementia. Further research focusing on those groups would be useful to have a more comprehensive view of the care trajectories of the older population.

\section{Conclusion}

Oldest-old people in China follow divergent trajectories of care utilisation, and the distribution of informal care provided by (grand)children in the older population is highly uneven. Persistent inequality in care utilisation and caregiving responsibilities have concerning consequences for the adequacy of care for older people and the wellbeing of caregivers in the long run, which warrant continued government interventions. Understanding the heterogeneity in, and the predictors of care trajectories is a crucial step that is of great value because it prospectively informs the design of longterm policy and the provision of government support that aim to bridge the looming gap between care demand and supply in China. The latent trajectory analysis and the machine learning approach prove to be effective tools to process large-scale longitudinal data and recognise the 'signal' with regard to care utilisation from a massive amount of information. Prediction models based on those methods have great potential for applications in practice, which will promote personalised care planning and the equitable allocation of care resources. 


\section{References}

Anderson, R., \& Newman, J. F. (2005). Societal and individual determinants of medical care utilization in the United States. Milbank Quarterly, 83(4), 1-28. https://doi.org/10.1111/j.14680009.2005.00428.x.

Burchardt, T., Jone, E., \& Obolenskaya, P. (2018). Formal and informal long-term care in the community: interlocking or incoherent systems? Journal of Social Policy, 47(3), 479-503. https://doi.org/10.1017/S0047279417000903.

Carmichael, F., \& Charles, S. (2003). The opportunity costs of informal care: does gender matter? Journal of health economics, 22(5), 781-803. https://doi.org/10.1016/S0167-6296(03)00044-4.

Casanova, R., Saldana, S., Lutz, M. W., Plassman, B. L., Kuchibhatla, M., \& Hayden, K. M. (2018). Investigating predictors of cognitive decline using machine learning. Journals of Gerontology: Psychological Sciences(April), 1-10. https://doi.org/10.1093/geronb/gby054.

Colombo, F., Llena-Nozal, A., Mercier, J., \& Tjadens, F. (2011). Help wanted? Providing and paying for long-term care. Paris: OECD Publishing. Available at: http://www.oecd.org/els/healthsystems/help-wanted-9789264097759-en.htm. Accessed on 28 February 2019.

Facal, D., Valladares - Rodriguez, S., Lojo - Seoane, C., Pereiro, A. X., Anido - Rifon, L., \& Juncos - Rabadán, O. (2019). Machine learning approaches to studying the role of cognitive reserve in conversion from mild cognitive impairment to dementia. International journal of geriatric psychiatry, 34(7), 941-949. https://doi.org/10.1002/gps.5090.

Feng, Z., Liu, C., Guan, X., \& Mor, V. (2012). China's rapidly aging population creates policy challenges in shaping a viable long-term care system. Health Affairs, 31(12), 2764-2773. http://doi.org/10.1377/hlthaff.2012.0535.

García-Gómez, P., Hernández-Quevedo, C., Jiménez-Rubio, D., \& Oliva-Moreno, J. (2015). Inequity in long-term care use and unmet need: two sides of the same coin. Journal of health economics, 39(January), 147-158. https://doi.org/10.1016/i.jhealeco.2014.11.004.

Grundy, E., \& Read, S. (2012). Social contacts and receipt of help among older people in England: are there benefits of having more children? Journals of Gerontology Series B: Psychological Sciences and Social Sciences, 67(6), 742-754. https://doi.org/10.1093/geronb/gbs082.

Hanaoka, C., \& Norton, E. C. (2008). Informal and formal care for elderly persons: How adult children's characteristics affect the use of formal care in Japan. Social Science \& Medicine, 67(6), 1002-1008. https://doi.org/10.1016/j.socscimed.2008.05.006.

Hastie, T., Tibshirani, R., \& Friedman, J. (2009). The Elements of Statistical Learning. New York: Springer.

Haviland, A. M., Jones, B. L., \& Nagin, D. S. (2011). Group-based trajectory modeling extended to account for nonrandom participant attrition. Sociological Methods \& Research, 40(2), 367-390. https://doi.org/10.1177/0049124111400041. 
Hu, B., Li, B., Wang, J., \& Shi, C. (2020). Home and community care for older people in urban China: Receipt of services and sources of payment. Health \& Social Care in the Community, 28(1), 225-235. https://doi.org/10.1111/hsc.12856.

Hu, B., \& Li, L. (2020). The protective effects of informal care receipt against the progression of functional limitations among Chinese older people. The Journals of Gerontology: Series B, 75(5), 1030-1041. https://doi.org/10.1093/geronb/gby107.

Hu, B., \& Ma, S. (2018). Receipt of informal care in the Chinese older population. Ageing and Society, 38(4), 766-793. https://doi.org/10.1017/S0144686X16001318.

Hu, B., \& Wang, J. (2019). Unmet long-term care needs and depression: the double disadvantage of community-dwelling older people in rural China Health and Social Care in the Community, 27(1), 126-138. https://doi.org/10.1111/hsc. 12630 .

Kemper, P. (1992). The Use of Formal and Informal Home Care by the Disabled Elderly. Health Services Research, 27(4), 421-451.

Kuhn, M., \& Johnson, K. (2013). Applied predictive modeling. New York: Springer.

Li, L. W. (2005). Longitudinal changes in the amount of informal care among publicly paid home care recipients. The Gerontologist, 45(4), 465-473. https://doi.org/10.1093/geront/45.4.465.

Liu, T., \& Sun, L. (2016). Pension reform in China. Journal of aging \& social policy, 28(1), 15-28. https://doi.org/10.1080/08959420.2016.1111725.

Miller, B., \& McFall, S. (1991). Stability and change in the informal task support network of frail older persons. The Gerontologist, 31(6), 735-745. https://doi.org/10.1093/geront/31.6.735.

Nagin, D. S. (2005). Group-based modeling of development. London: Harvard University Press.

Peng, R., Wu, B., \& Ling, L. (2015). Undermet needs for assistance in personal activities of daily living among community-dwelling oldest old in China from 2005 to 2008. Research on Aging, 37(2), 148-170. https://doi.org/10.1177/0164027514524257.

Pickard, L., Wittenberg, R., Comas-Herrera, A., Davies, B., \& Darton, R. (2000). Relying on Informal Care in the New Century? Informal Care for Elderly in England to 2031. Ageing and Society, 20(6), 745-772.

Robards, J., Vlachantoni, A., Evandrou, M., \& Falkingham, J. (2015). Informal caring in England and Wales-Stability and transition between 2001 and 2011. Advances in Life Course Research, 24, 21-33. https://doi.org/10.1016/j.alcr.2015.04.003.

Rodrigues, R., Ilinca, S., \& Schmidt, A. E. (2018). Income - rich and wealth - poor? The impact of measures of socio - economic status in the analysis of the distribution of long - term care use among older people. Health economics, 27(3), 637-646. https://doi.org/10.1002/hec.3607. 
Shi, C., \& Hu, B. (2020). Preferences for formal social care in rural and urban China: Evidence from a national survey. Journal of Gerontological Social Work, 63(1-2), 19-40.

https://doi.org/10.1080/01634372.2019.1709246.

Sole-Auro, A., \& Crimmins, E. M. (2014). Who cares? A comparison of informal and formal care provision in Spain, England and the USA. Ageing \& Society, 34(3), 495-517.

Spector, W. D., \& Fleishman, J. A. (1998). Combining activities of daily living with instrumental activities of daily living to measure functional disability. The Journals of Gerontology Series B: Psychological Sciences and Social Sciences, 53(1), S46-S57.

https://doi.org/10.1093/geronb/53B.1.S46.

Suanet, B., Van Groenou, M. B., \& Van Tilburg, T. (2012). Informal and Formal Home-Care Use among Older Adults in Europe: Can Cross-National Differences be Explained by Societal Context and Composition? Ageing and Society, 32(3), 491-515. https://doi.org/10.1017/S0144686X11000390.

United Nations. (2019). World population prospects 2019. New York: United Nations.

Van Houtven, C. H., \& Norton, E. C. (2004). Informal care and health care use of older adults. Journal of Health Economics, 23, 1159-1180.

Vlachantoni, A. (2019). Unmet need for social care among older people. Ageing \& Society, 39(4), 657-684. https://doi.org/10.1017/S0144686X17001118.

Vlachantoni, A., Shaw, R. J., Evandrou, M., \& Falkingham, J. (2015). The determinants of receiving social care in later life in England. Ageing and Society, 35(2), 321-345.

https://doi.org/10.1017/S0144686X1300072X.

Wang, J., Yang, Q., \& Wu, B. (2020). Effects of Care Arrangement on the Age of Institutionalization among Community-dwelling Chinese Older Adults. Journal of Aging \& Social Policy, 1-16.

https://doi.org/10.1080/08959420.2020.1726720.

Weiss, C. O., Gonzalez, H. M., Kabeto, M. U., \& Langa, K. M. (2005). Differences in Amount of Informal Care Received by Non-Hispanic Whites and Latinos in a Nationally Representative Sample of Older American. Journal of American Geriatrics Society, 53, 146-151.

https://doi.org/10.1111/j.1532-5415.2005.53027.x.

Wittenberg, R., Hu, B., Jagger, C., Kingston, A., Knapp, M., Comas-Herrera, A., King, D., Rehill, A., $\&$ Banerjee, S. (2020). Projections of care for older people with dementia in England: 2015 to 2040. Age and Ageing, 49(2), 264-269.

Wolf, D. A. (2014). Getting help from others: The effects of demand and supply. Journals of Gerontology Series B: Psychological Sciences and Social Sciences, 69(Suppl_1), S59-S64. https://doi.org/10.1093/geronb/gbu122.

World Health Organisation. (2015). World report on ageing and health. Geneva: World Health Organisation. 
Yang, W., Jingwei He, A., Fang, L., \& Mossialos, E. (2016). Financing institutional long-term care for the elderly in China: a policy evaluation of new models. Health Policy and Planning, 31(10), 1391-1401. https://doi.org/10.1093/heapol/czw081.

Zeng, Y., Vaupel, J. W., Xiao, Z., Zhang, C., \& Liu, Y. (2002). Sociodemographic and health profiles of the oldest old in China. Population and Development Review, 28(2), 251-273.

https://doi.org/10.1111/j.1728-4457.2002.00251.x.

Zhan, H. J., \& Montgomery, R. J. V. (2003). Gender and Elder Care in China: The Influence of Filial Piety and Structural Constraints. Gender and Society, 17(2), 209-229.

https://doi.org/10.1177/0891243202250734.

Zhu, H. (2015). Unmet needs in long-term care and their associated factors among the oldest old in China. BMC geriatrics, 15(1), 46.

Zimmer, Z., Martin, L. G., Nagin, D. S., \& Jones, B. L. (2012). Modeling disability trajectories and mortality of the oldest-old in China. Demography, 49(1), 291-314. https://doi.org/10.1007/s13524$\underline{011-0075-7 .}$. 
Table 1 Results of latent trajectory analysis with non-random attrition (three-group model)

\begin{tabular}{|c|c|c|c|}
\hline & $\begin{array}{c}\text { Trajectory } 1 \\
\text { (Low intensity) }\end{array}$ & $\begin{array}{c}\text { Trajectory } 2 \\
\text { (Increased intensity) }\end{array}$ & $\begin{array}{c}\text { Trajectory } 3 \\
\text { (High intensity) }\end{array}$ \\
\hline Number of people in 2005 & $8,834(85.8 \%)$ & $1,027(10.0 \%)$ & $431(4.2 \%)$ \\
\hline \multicolumn{4}{|l|}{ Average hours of care per week } \\
\hline 2005 (wave 1) & 6.8 & 29.1 & 121.4 \\
\hline 2008 (wave 2) & 1.5 & 38.4 & 165.3 \\
\hline 2011 (wave 3) & 5.2 & 75.2 & 168 \\
\hline 2014 (wave 4) & 8.4 & 107.5 & 168 \\
\hline \multicolumn{4}{|c|}{ Coefficients (standard error): main model } \\
\hline Constant & $-6.9(7.2)$ & $-31.2 * * *(8.9)$ & $85.2(184.7)$ \\
\hline Linear term & $-62.7 * * *(8.3)$ & $1.9(10.0)$ & $27.2(280.4)$ \\
\hline Quadratic term & $13.9 * * *(1.7)$ & $9.2 * * *(3.7)$ & $25.1(95.1)$ \\
\hline \multicolumn{4}{|c|}{ Coefficients (standard error): attrition model } \\
\hline Constant & $-6.6 * * *(0.4)$ & $-2.8 * * *(0.6)$ & $-6.7 *(2.9)$ \\
\hline Lagged response & $0.03 * * *(0.01)$ & $0.001(0.001)$ & $0.03 * * *(0.004)$ \\
\hline Age in 2005 & $0.1 * * *(0.004)$ & $0.04 * * *(0.01)$ & $0.03(0.03)$ \\
\hline $\mathrm{ADL}$ in 2005 & $0.6 *(0.2)$ & $0.5^{* * *}(0.1)$ & $1.4^{* * *}(0.4)$ \\
\hline IADL in 2005 & $0.6^{* * *}(0.06)$ & $-0.1(0.07)$ & $0.2(0.4)$ \\
\hline \multicolumn{4}{|l|}{ Model adequacy } \\
\hline $\begin{array}{l}\text { Average posterior probability } \\
\text { (APP) }\end{array}$ & 0.73 & 0.76 & 0.80 \\
\hline $\begin{array}{l}\text { Odds of correct classification } \\
\text { (OCC) }\end{array}$ & 5.3 & 6.5 & 8.0 \\
\hline
\end{tabular}

Note: The average hours of care in 2014 for people in the high intensity trajectory were extrapolated from the model as none in this group survived or stayed in the survey until $2014 ;{ }^{*} \mathrm{p}<0.05,{ }^{* *} \mathrm{p}<0.01$, $* * * \mathrm{p}<0.001$. 
Table 2 Sample characteristics in 2005 and univariate analysis of association with care trajectories

\begin{tabular}{|c|c|c|c|c|}
\hline Variables & Low intensity & $\begin{array}{c}\text { Increased } \\
\text { intensity }\end{array}$ & High intensity & $\begin{array}{c}\text { Test of } \\
\text { association }\end{array}$ \\
\hline \multicolumn{5}{|c|}{ Means or proportions } \\
\hline \multicolumn{5}{|l|}{ Need factors } \\
\hline ADL score & 0.20 & 0.41 & 0.89 & $\rho=0.29 * * *$ \\
\hline IADL score & 0.87 & 1.27 & 1.72 & $\rho=0.24 * * *$ \\
\hline Number of diseases & 0.84 & 1.07 & 1.30 & $\rho=0.08 * * *$ \\
\hline \multicolumn{5}{|l|}{ Enabling factors } \\
\hline \multicolumn{5}{|l|}{ Living arrangements } \\
\hline Living with (grand)children & $72.3 \%$ & $81.6 \%$ & $91.4 \%$ & \\
\hline Living with spouse only & $17.7 \%$ & $11.2 \%$ & $5.1 \%$ & \\
\hline Living alone/with relatives & $10.0 \%$ & $7.2 \%$ & $3.5 \%$ & $\chi^{2}=113.0^{* * *}$ \\
\hline \multicolumn{5}{|l|}{ Number of sons } \\
\hline None & $16.6 \%$ & $16.7 \%$ & $18.3 \%$ & \\
\hline $1-3$ sons & $71.8 \%$ & $70.7 \%$ & $71.2 \%$ & \\
\hline 4 or more sons & $11.7 \%$ & $12.7 \%$ & $10.4 \%$ & $\chi^{2}=2.3$ \\
\hline \multicolumn{5}{|l|}{ Number of daughters } \\
\hline None & $23.0 \%$ & $22.4 \%$ & $19.0 \%$ & \\
\hline 1-3 daughters & $67.6 \%$ & $65.4 \%$ & $71.7 \%$ & \\
\hline 4 or more daughters & $9.4 \%$ & $12.2 \%$ & $9.3 \%$ & $\chi^{2}=11.9 *$ \\
\hline \multicolumn{5}{|l|}{ Demographic factors } \\
\hline \multicolumn{5}{|l|}{ Age } \\
\hline $80-89$ & $38.3 \%$ & $30.4 \%$ & $17.9 \%$ & \\
\hline $90-99$ & $37.1 \%$ & $37.2 \%$ & $35.5 \%$ & \\
\hline $100+$ & $24.6 \%$ & $32.4 \%$ & $46.6 \%$ & $\chi^{2}=151.3 * * *$ \\
\hline \multicolumn{5}{|l|}{ Gender } \\
\hline Male & $41.1 \%$ & $27.3 \%$ & $25.8 \%$ & \\
\hline Female & $58.9 \%$ & $72.7 \%$ & $74.2 \%$ & $\chi^{2}=108.0^{* * *}$ \\
\hline \multicolumn{5}{|l|}{ Residence } \\
\hline Urban China & $42.6 \%$ & $48.6 \%$ & $61.7 \%$ & \\
\hline Rural China & $57.4 \%$ & $51.4 \%$ & $38.3 \%$ & $\chi^{2}=71.0 * * *$ \\
\hline \multicolumn{5}{|l|}{ Socioeconomic factors } \\
\hline \multicolumn{5}{|l|}{ Financial status } \\
\hline Poorer & $18.1 \%$ & $19.9 \%$ & $19.9 \%$ & \\
\hline Average & $65.6 \%$ & $64.3 \%$ & $62.8 \%$ & \\
\hline Richer & $16.3 \%$ & $15.8 \%$ & $17.3 \%$ & $\chi^{2}=3.4$ \\
\hline Income per capita & 7.92 & 8.00 & 8.26 & $\rho=0.08 * * *$ \\
\hline \multicolumn{5}{|l|}{ Formal education } \\
\hline No & $68.6 \%$ & $74.6 \%$ & $72.8 \%$ & \\
\hline Yes & $31.4 \%$ & $25.4 \%$ & $27.2 \%$ & $\chi^{2}=17.8^{* * *}$ \\
\hline \multicolumn{5}{|l|}{ Primary carer } \\
\hline No ADL limitations/carers & $71.4 \%$ & $43.5 \%$ & $12.5 \%$ & \\
\hline (Grand)children/spouse & $25.2 \%$ & $52.4 \%$ & $81.2 \%$ & \\
\hline Other caregivers & $3.4 \%$ & $4.1 \%$ & $6.3 \%$ & $\chi^{2}=923.1 * * *$ \\
\hline Sample size & & 10,292 & & \\
\hline
\end{tabular}

Notes: $\rho$ denotes the Spearman correlation coefficient; $\chi^{2}$ denotes the Pearson's Chi square statistic; ${ }^{*} \mathrm{p}<0.05,{ }^{* *} \mathrm{p}<0.01,{ }^{* * *} \mathrm{p}<0.001$; Other caregivers include relatives or paid helpers. 
Table 3 Model performance in predicting trajectories of informal care intensity

\begin{tabular}{|c|c|c|c|c|}
\hline \multicolumn{5}{|l|}{ Base case analysis } \\
\hline & LR & $\mathrm{RF}$ & SVM & ANN \\
\hline \multicolumn{5}{|c|}{ Increased vs low intensity } \\
\hline Accuracy & 0.62 & 0.64 & 0.62 & 0.63 \\
\hline Sensitivity & 0.63 & 0.66 & 0.64 & 0.72 \\
\hline Specificity & 0.61 & 0.62 & 0.59 & 0.54 \\
\hline \multicolumn{5}{|c|}{ High vs low intensity } \\
\hline Accuracy & 0.79 & 0.79 & 0.79 & 0.79 \\
\hline Sensitivity & 0.82 & 0.82 & 0.84 & 0.81 \\
\hline Specificity & 0.76 & 0.76 & 0.73 & 0.77 \\
\hline \multicolumn{5}{|c|}{ High vs increased intensity } \\
\hline Accuracy & 0.68 & 0.69 & 0.69 & 0.68 \\
\hline Sensitivity & 0.67 & 0.71 & 0.69 & 0.69 \\
\hline Specificity & 0.69 & 0.66 & 0.69 & 0.67 \\
\hline \multicolumn{5}{|c|}{ Accuracy of RF classifier: model optimisation } \\
\hline & 10-fold Cross-validation & Bootstrap & 10-fold CV & id search \\
\hline Increased vs low & 0.64 & 0.64 & \multicolumn{2}{|c|}{0.65} \\
\hline High vs low & 0.80 & 0.80 & \multicolumn{2}{|c|}{0.81} \\
\hline High vs increased & 0.70 & 0.69 & \multicolumn{2}{|c|}{0.71} \\
\hline
\end{tabular}


Table 4 Relative importance of predictors in contributing to prediction accuracy in the RF classifier

\begin{tabular}{llllll}
\hline \multicolumn{2}{l}{ Increased vs low intensity } & \multicolumn{2}{l}{ High vs low intensity } & \multicolumn{2}{l}{ High vs increased intensity } \\
\hline Predictor & Importance & Predictor & Importance & Predictor & Importance \\
\hline ADL & 100 & GCSC & 100 & ADL & 100 \\
IADL & 81 & IADL & 96 & Income & 73 \\
GCSC & 62 & ADL & 67 & IADL & 39 \\
Disease & 15 & LA & 14 & Disease & 26 \\
Gender & 15 & Income & 20 & GCSC & 17 \\
Residence & 13 & Disease & 18 & Daughter & 16 \\
Income & 10 & Residence & 14 & LA & 10 \\
Age: $90-99$ & 8 & Age: $100+$ & 13 & Residence & 8 \\
FS: average & 8 & Gender & 11 & Age:90-99 & 7 \\
LA & 8 & Daughter & 5 & Age:100+ & 6 \\
FS: poorer & 7 & FS: average & 5 & FS: average & 5 \\
Daughter & 6 & Age: $90-99$ & 3 & Sons & 4 \\
Age: $100+$ & 4 & Education & 2 & Education & 3 \\
Other carers & 2 & Son & 1 & Gender & 3 \\
Education & 1 & Other carers & 0 & FS: poorer & 2 \\
Son & 0 & FS: poorer & 0 & Other carers & 0 \\
\hline
\end{tabular}

Note: GCSC: (grand)children/spouse carers; LA: living arrangements; FS: Self-reported financial status; $100=$ most important and $0=$ least important 


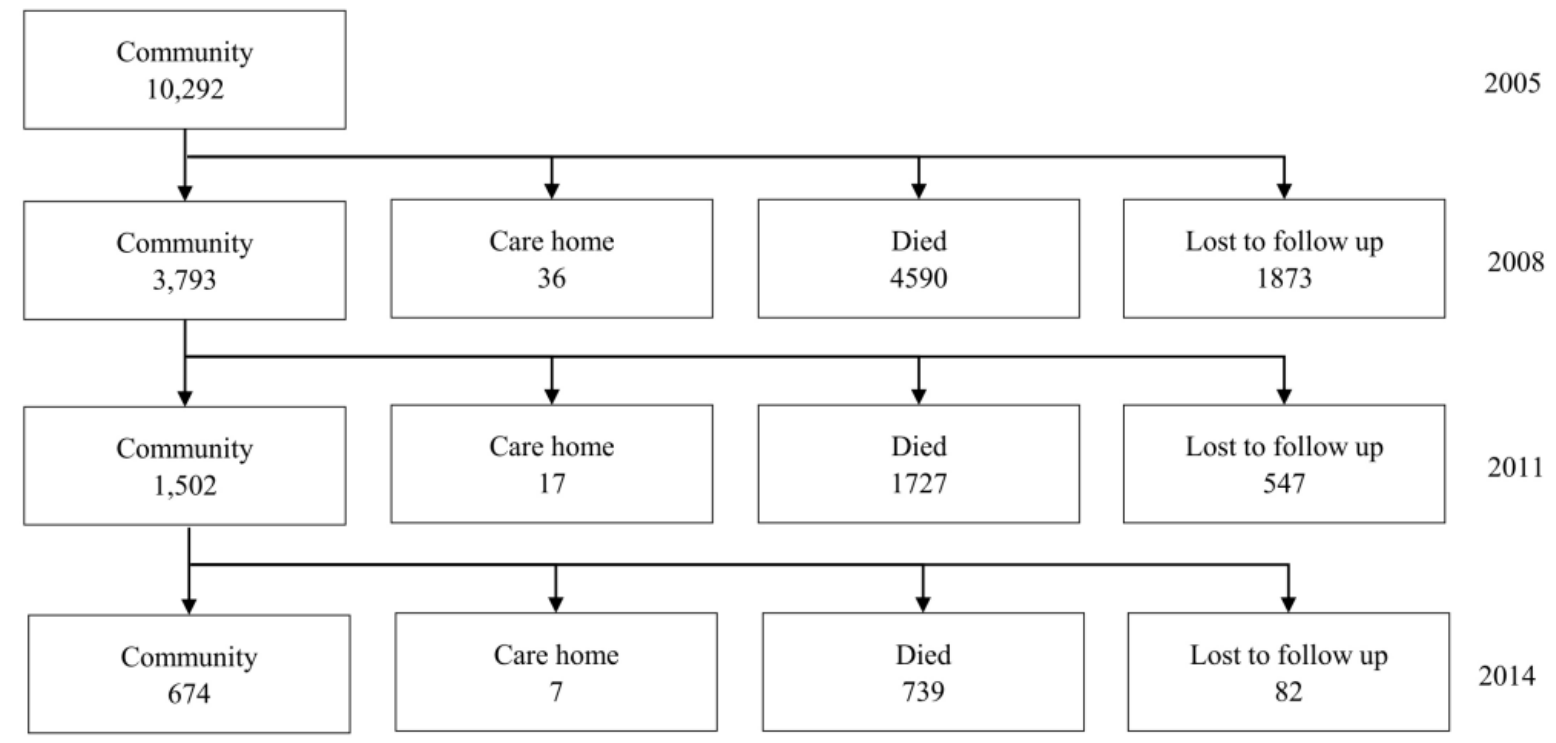

Figure 1 Four waves of the CLHLS data 


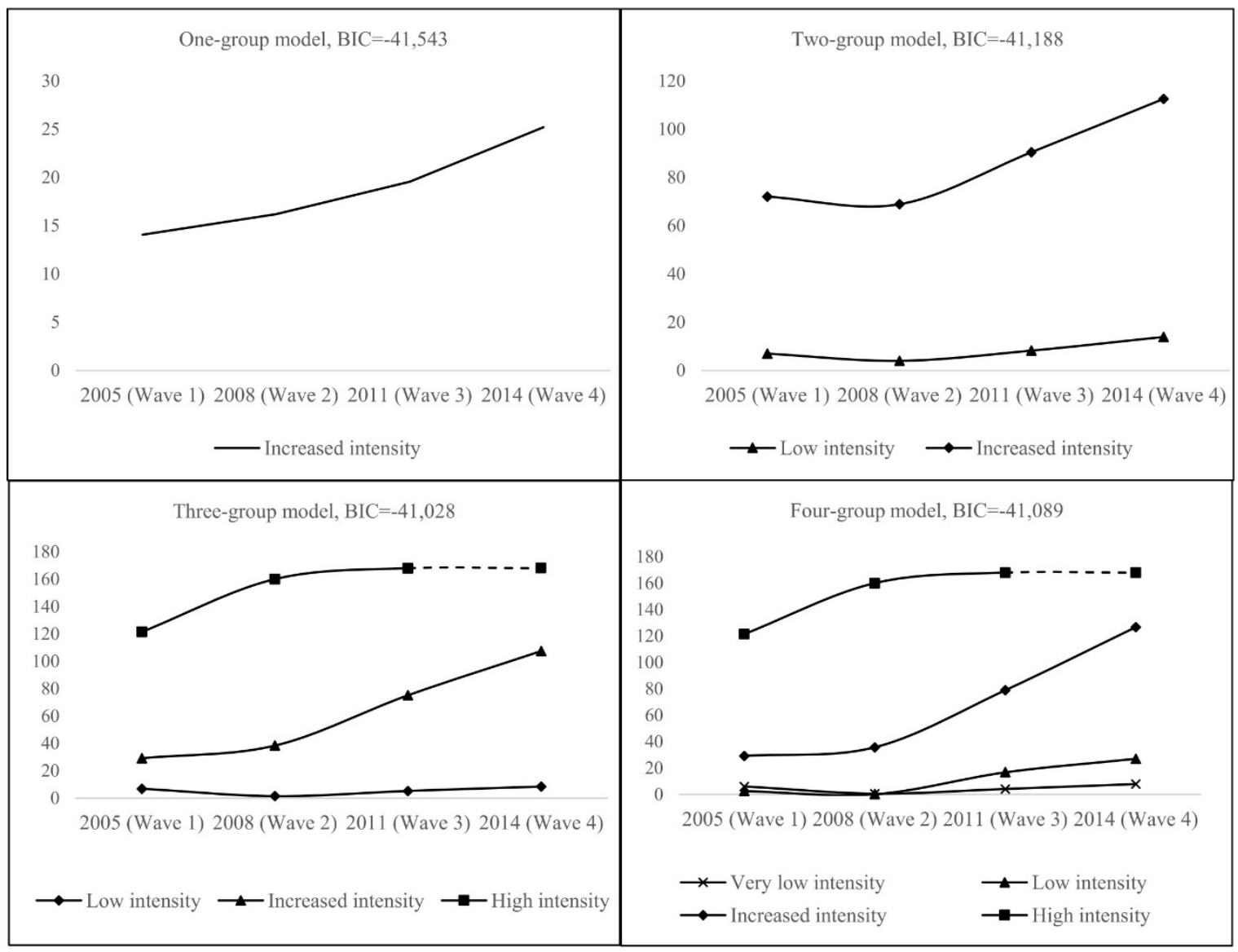

Figure 2 Latent trajectory analysis of informal care intensity

Note: The average hours of care in 2014 for people in the high intensity trajectory were extrapolated as none in this group survived or stayed in the survey until 2014; The three-group model has the best fit. 


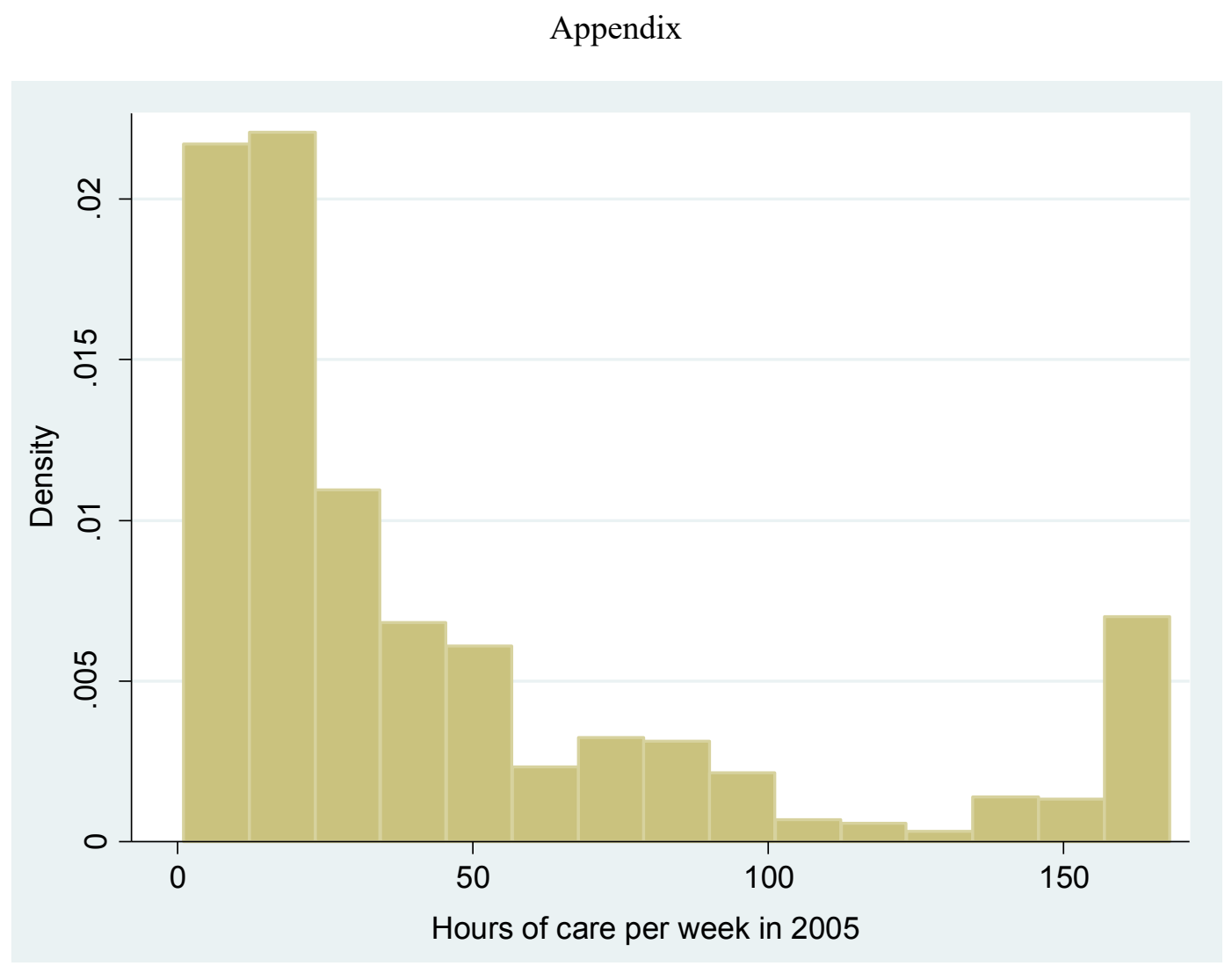

Figure A1 Weekly hours of care received from (grand)children in relation to ADL limitations in 2005 (care recipients only) 